\title{
SCIENCE AND ECONOMIC POLICY
}

\section{TAMAR DOLIDZE}

\section{PhD student}

Ivane Javakhishvili Tbilisi State University, Georgia

tamarduta@gmail.com

Abstract. In modern society human existence depends largely on scientific knowledge. Moreover, the world of our surroundings, events and the things we need are partly the result of scientific knowledge.

Science is a multifaceted social phenomenon. It is important to list some of the key science facets:

- vision function;

- Economic function of science;

- Science is also an element of culture.

In postindustrial society the fact that science and technology are intertwined is of particular importance since the development of technology without scientific knowledge is difficult to imagine. It should also be noted that science is at the core of everything in general: technology development, education, knowledge-based economics and human capital, published researches, published scientific articles, number of triad patents, innovations.

In view of the foregoing, the issue is of very urgent and pragmatic importance. It is therefore advisable to discuss Holden and Russellıs views on science.

Using the comparative analysis method, we will generalize three basic theories about science:

- The theory of evolutionary development of scientific knowledge (cumulative theory);

- The theory of revolutionary development of scientific knowledge;

- Conciliatory concept.

Using the comparative analysis method, we will identify one optimal theory and discuss it in the context of economic policy.

The context of the relationship between science and economic policy implies many problems. In this article, we will discuss the main problems of science field and find ways to solve them using economic policy.

We will analyze which economic policy approaches will be most optimal for solving problems in science (positive economics; normative economics; political economics;).

In our opinion, it is important to consider whether it is appropriate to include education and science in the third sector of the economy.

In the example of different countries of America we will analyze the statistics for 2010-2016. Specifically, what percentages of gross domestic product from the state budget are used to finance science and technology? Using the method of comparative analysis, we determine the trend formed in the given time series.

KEYWORDS: SCIENCE, ECONOMIC POLICY,FOURTH, FIFTH SECTOR OF ECONOMY, TRIAD PATENTS.

For citation: Dolidze, T. (2020). Science and Economic Policy. Globalization and Business, №9, pp. 179-186 (In Georgian). https://doi.org/10.35945/gb.2020.09.022 


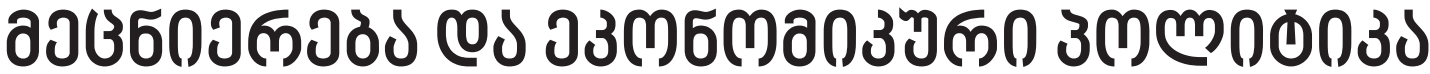

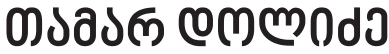

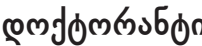

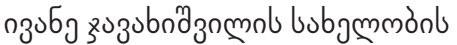

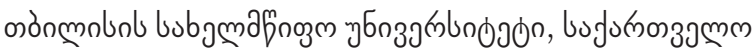

tamarduta@gmail.com

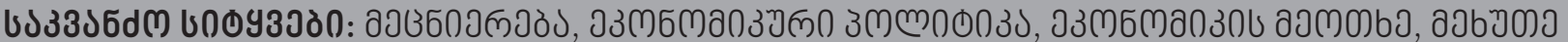

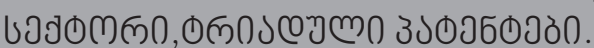

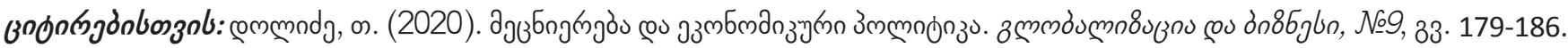
https://doi.org/10.35945/gb.2020.09.022

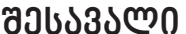

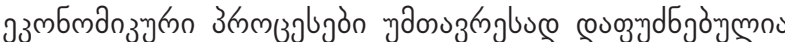

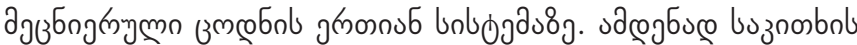

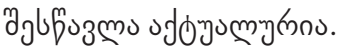

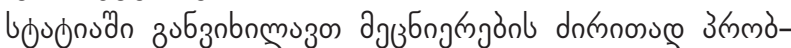

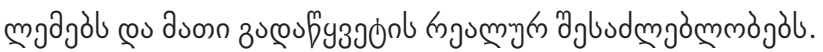

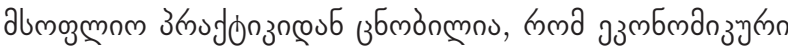

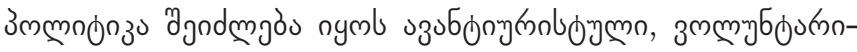

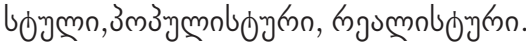

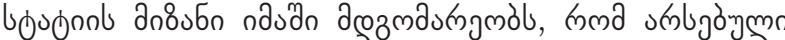

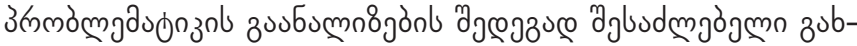
e el o

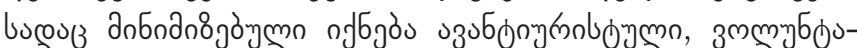

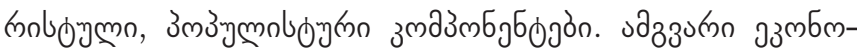

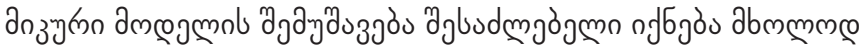

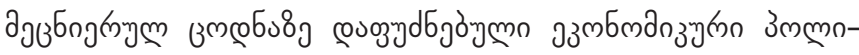

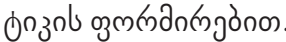

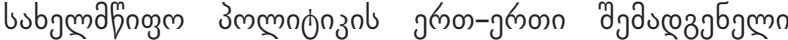

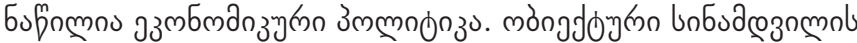

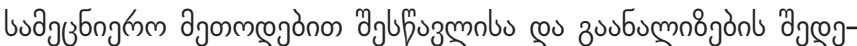

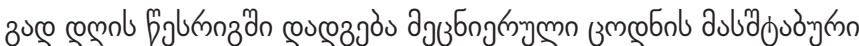

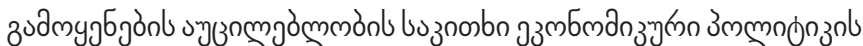

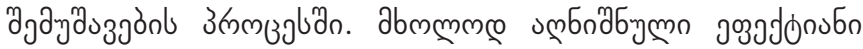

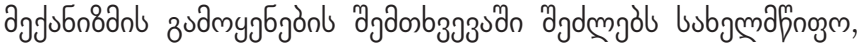

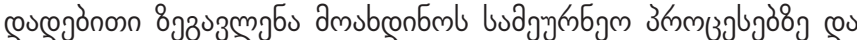

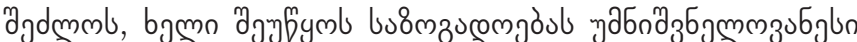

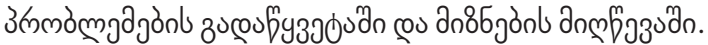

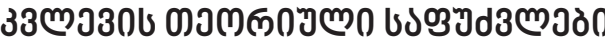

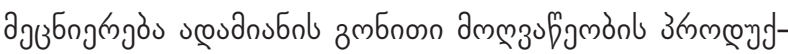

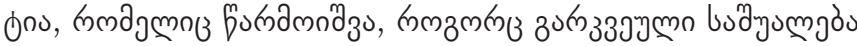

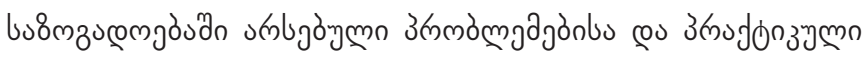

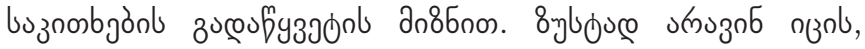

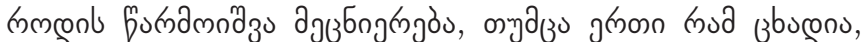

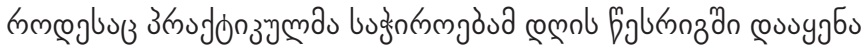

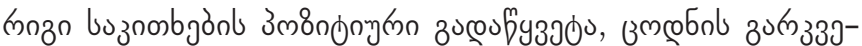

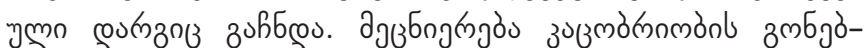

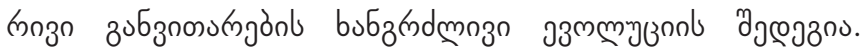

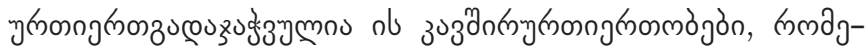

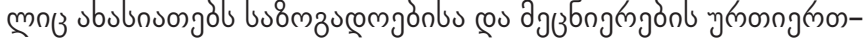

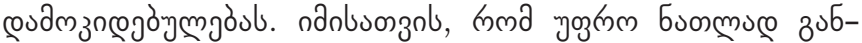

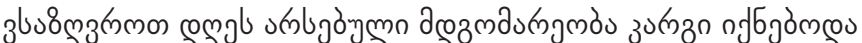

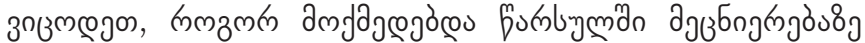

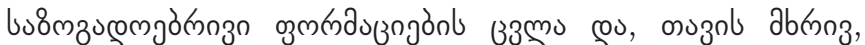

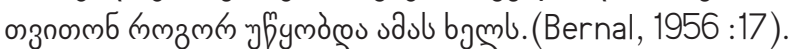

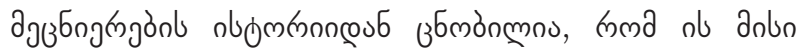

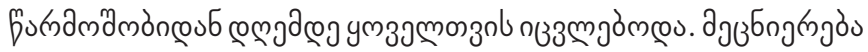

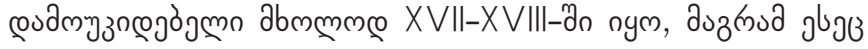

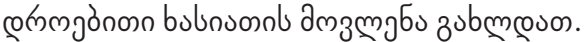

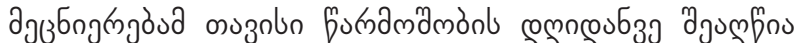

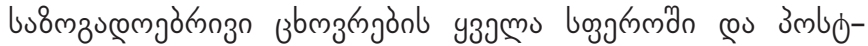

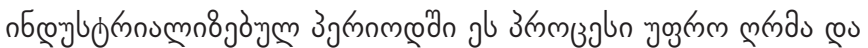

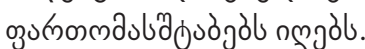

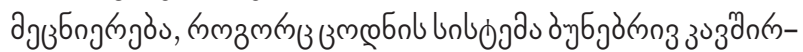

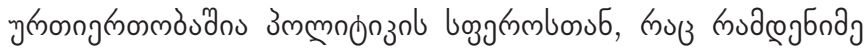

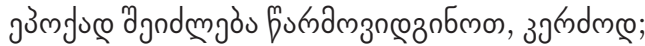

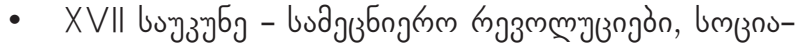

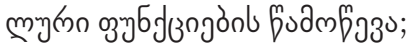

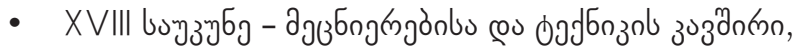

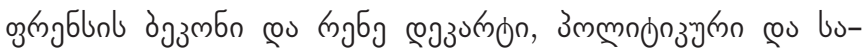

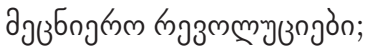

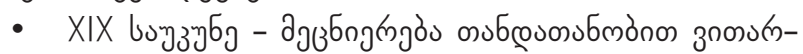

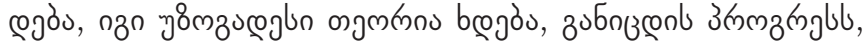

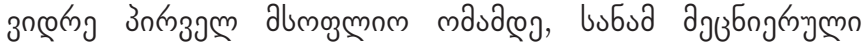




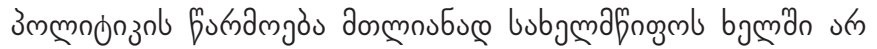
zомозо.

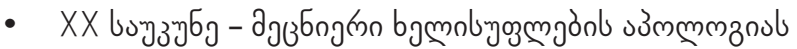

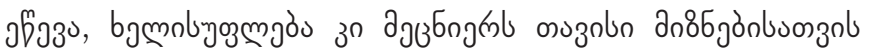

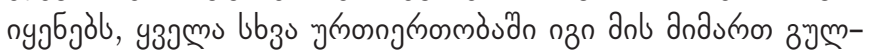
3

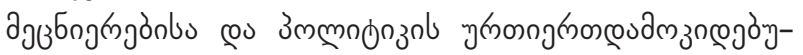

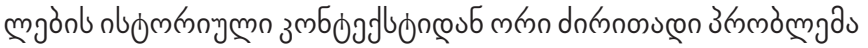

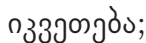

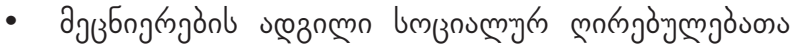
unbogas an;

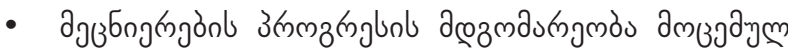
$30038 \mathrm{~g}$.

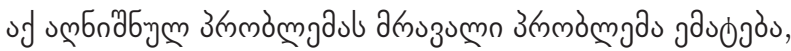

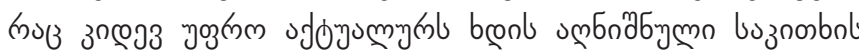

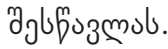

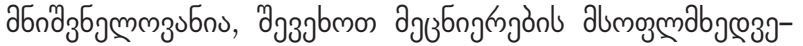

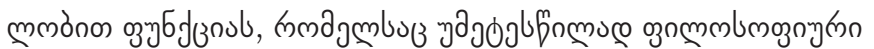

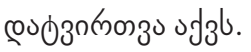

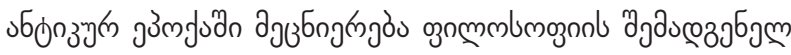

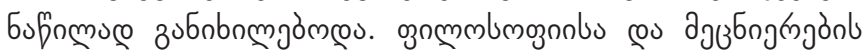

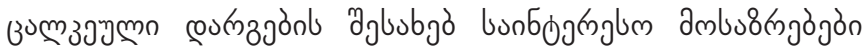

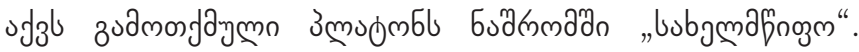

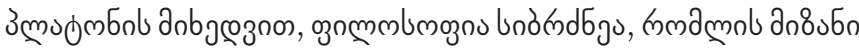

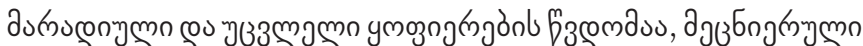

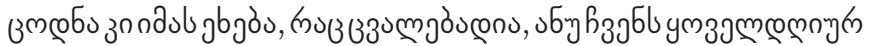

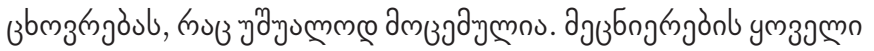

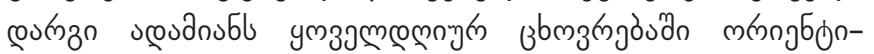

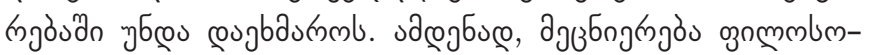

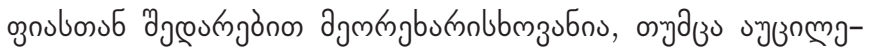

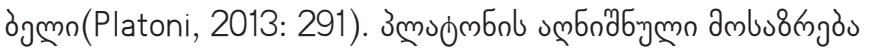

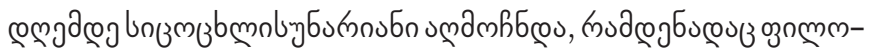

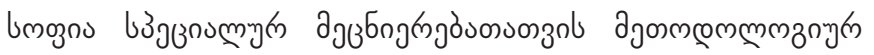

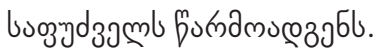

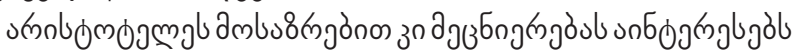

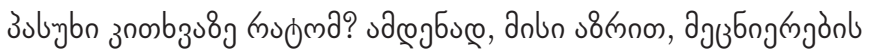

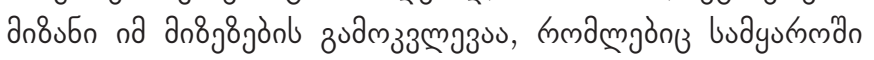

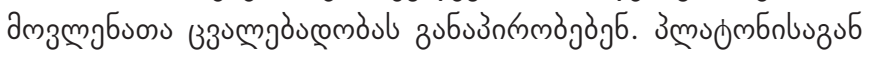

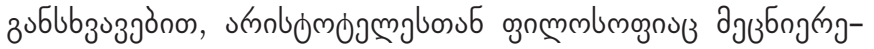
bos, bмmल

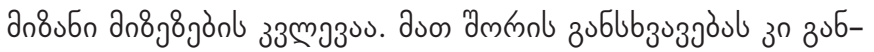

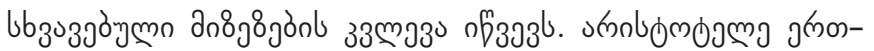

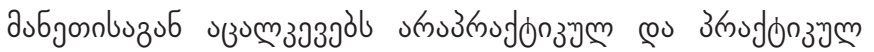

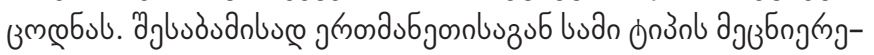

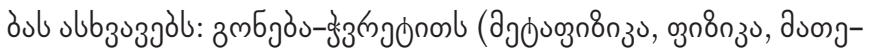

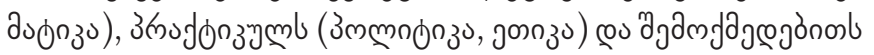

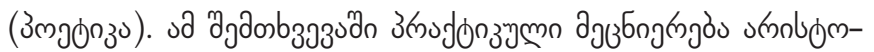

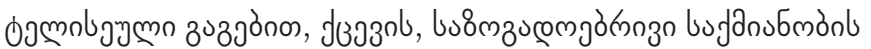

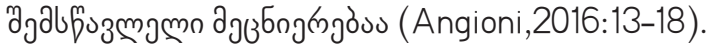

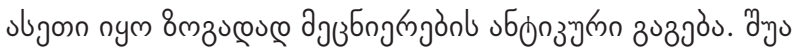

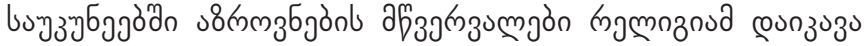
co ajo

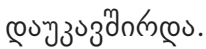

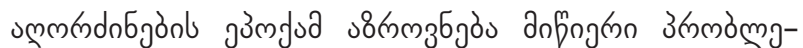

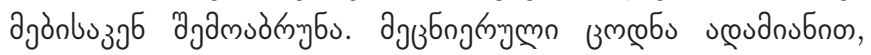

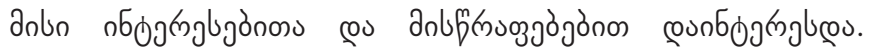

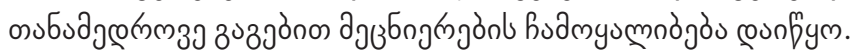

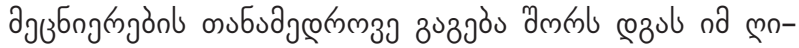

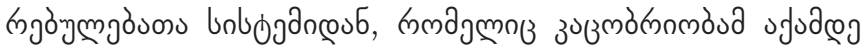

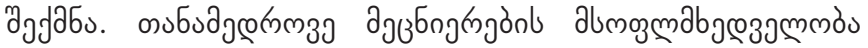

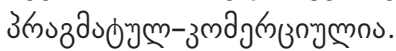

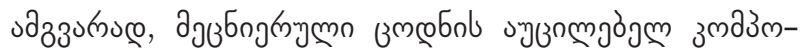

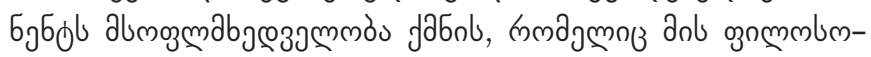

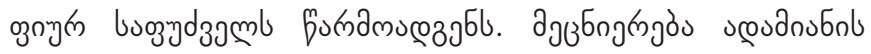

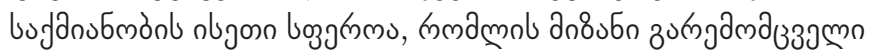

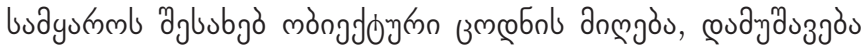

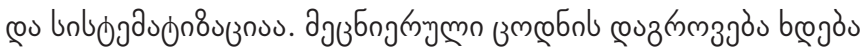

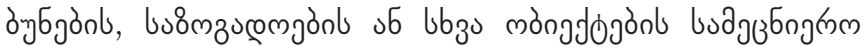

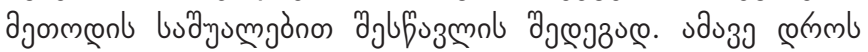

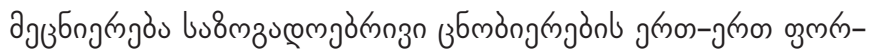

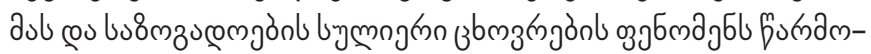
งезjбl.

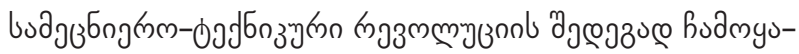

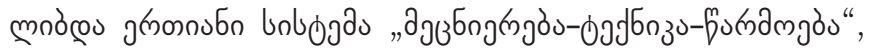

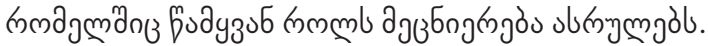

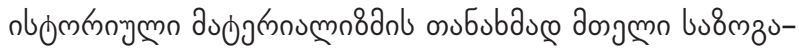

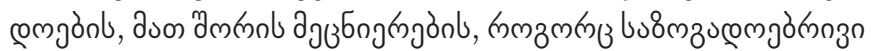

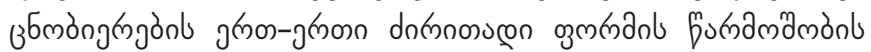

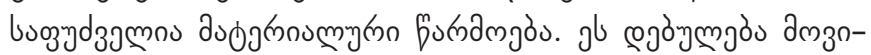

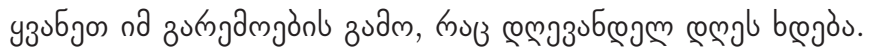

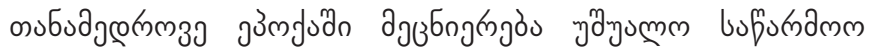
dumuse zucoundzu cou agzбngmym-

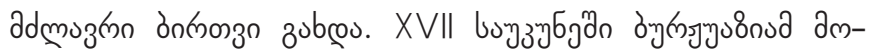

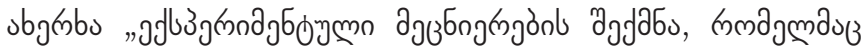

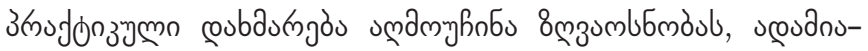

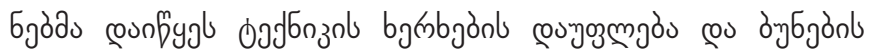

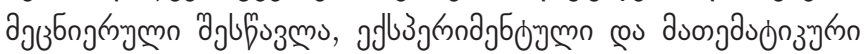

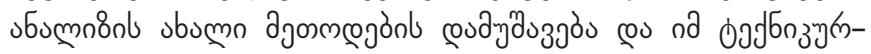

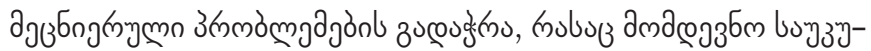

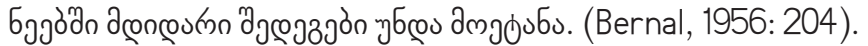

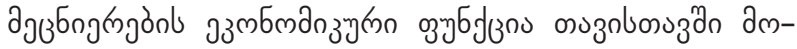

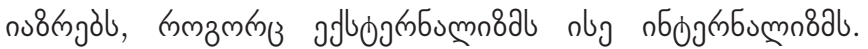

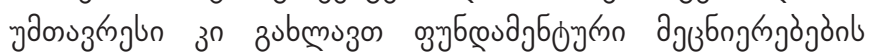

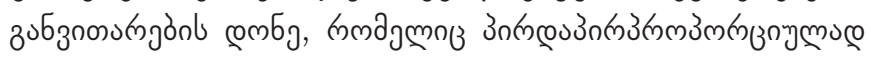
зuбbu8

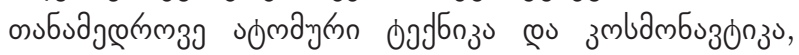

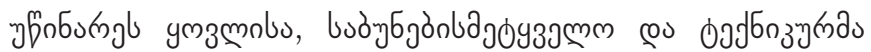

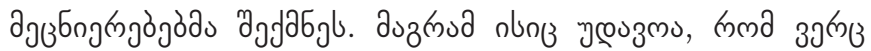

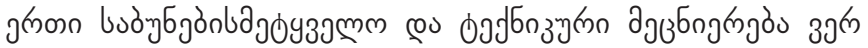

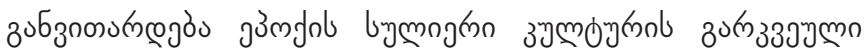




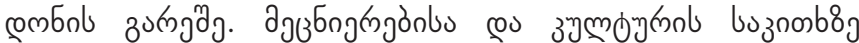

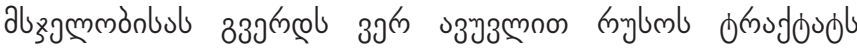

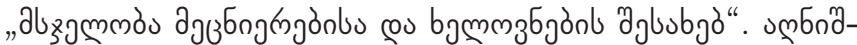

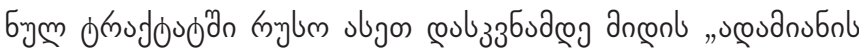

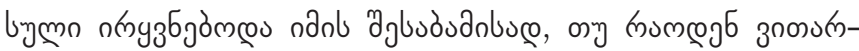

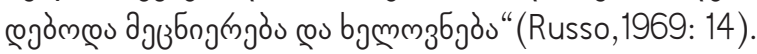

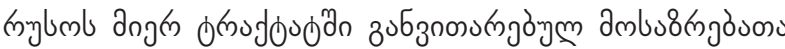

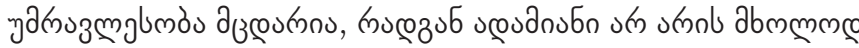

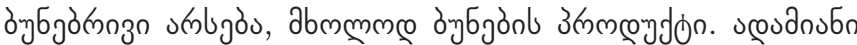

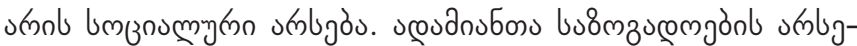

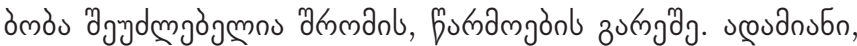

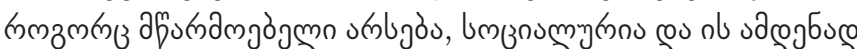

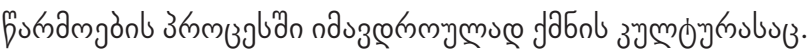

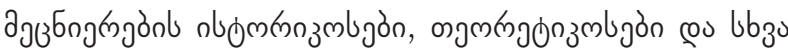

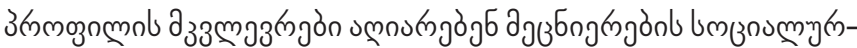

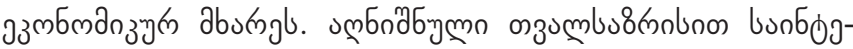

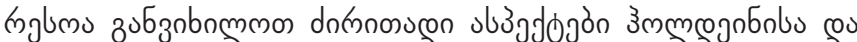

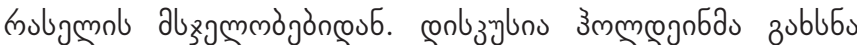
mszolu

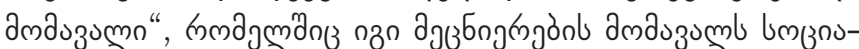

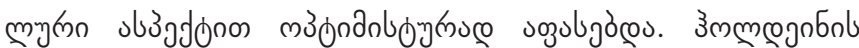

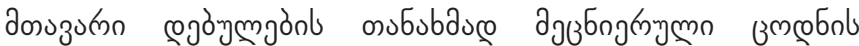

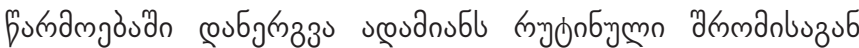

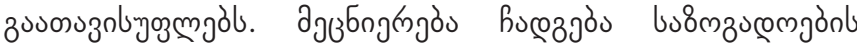

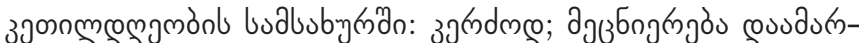

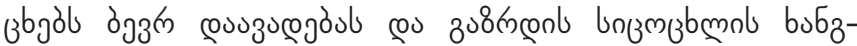

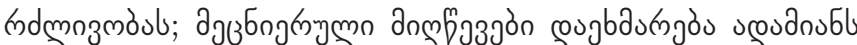

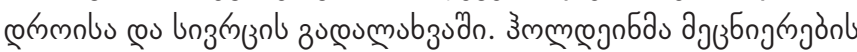

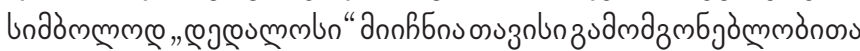
cos dudnjòjgmn zmбjònon. (Haldane, 1923:16-26).

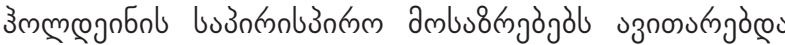

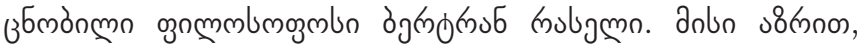

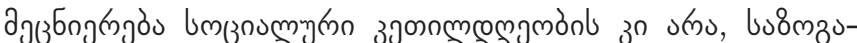

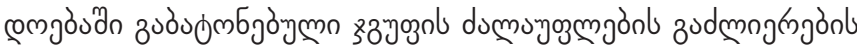

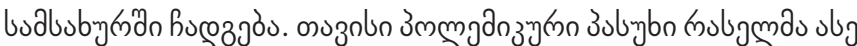

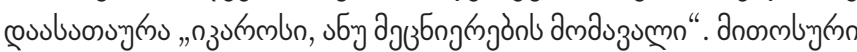

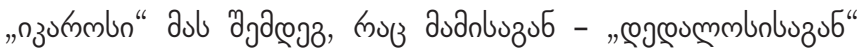

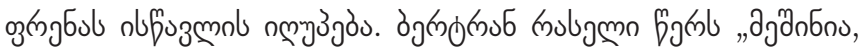

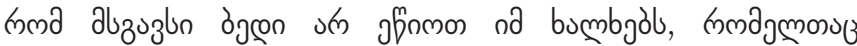

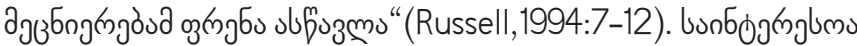
د а з з

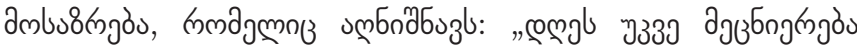

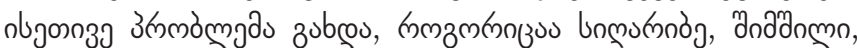

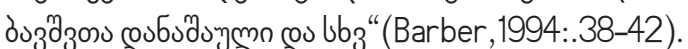

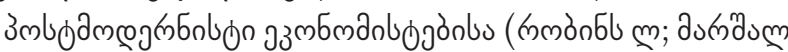

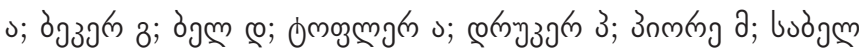

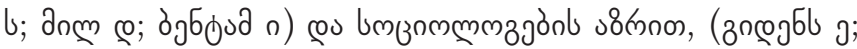

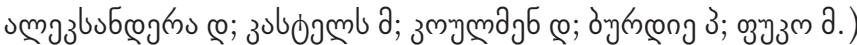

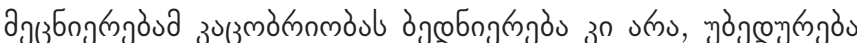

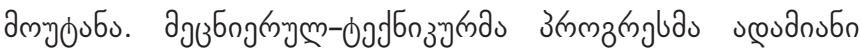

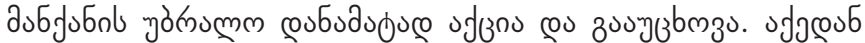

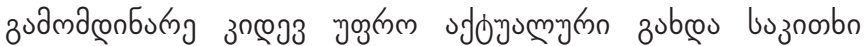

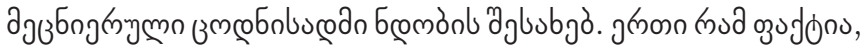

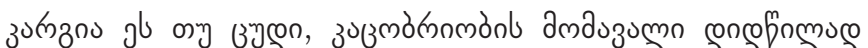

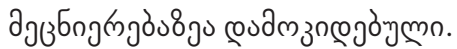

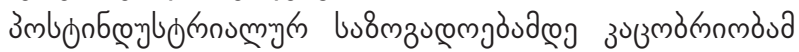

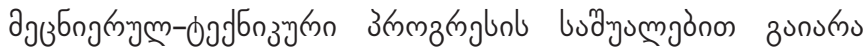

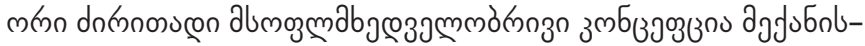

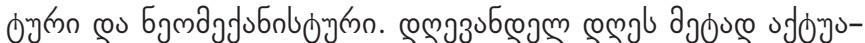
mynnn bmmnog

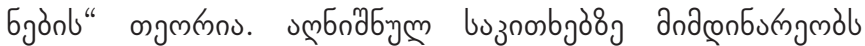

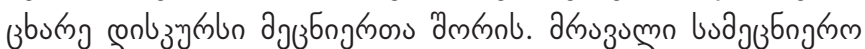

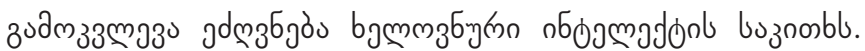

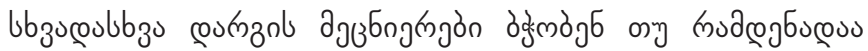

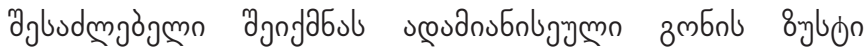

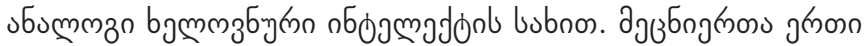

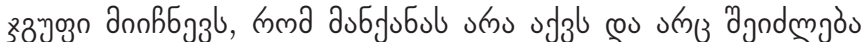

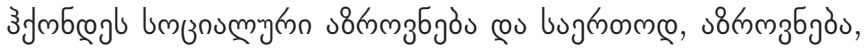
znбun

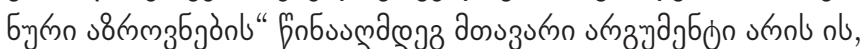
ஙma soిmm

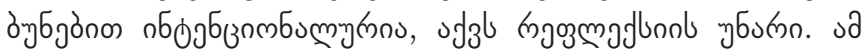

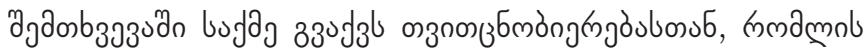

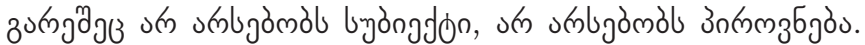

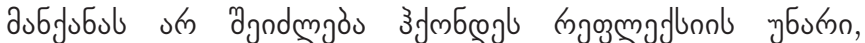

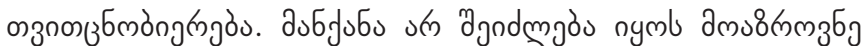

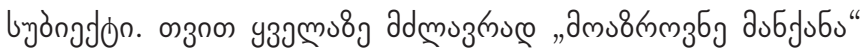

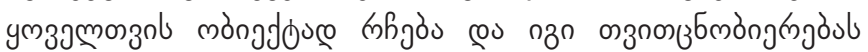

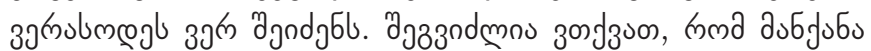

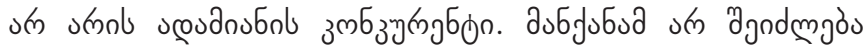

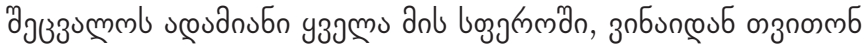

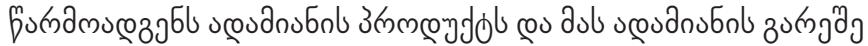

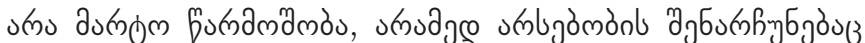

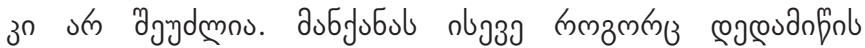

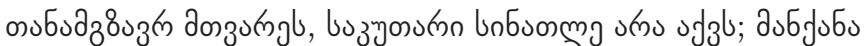

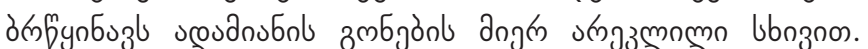
(Novik, 1963:136).

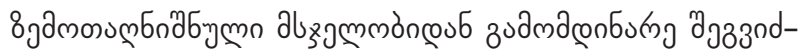

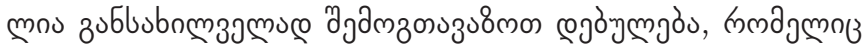

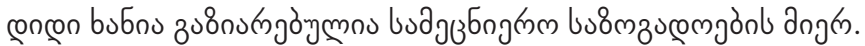

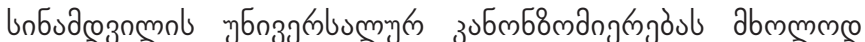

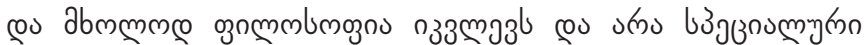

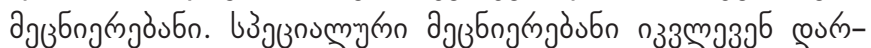

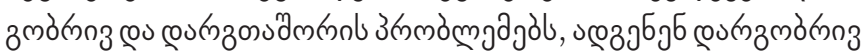

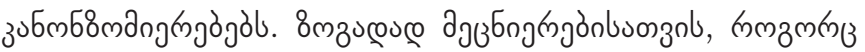

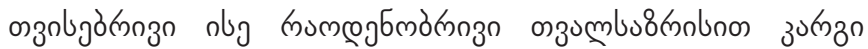

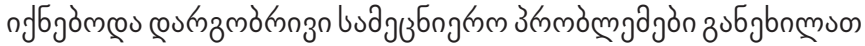

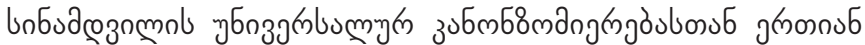

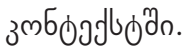

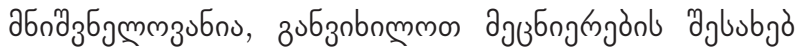




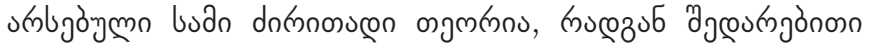

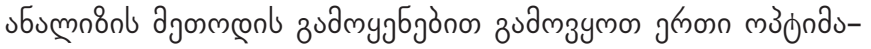
mynno ogmknos.

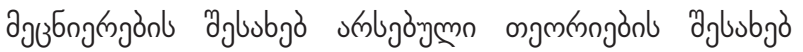

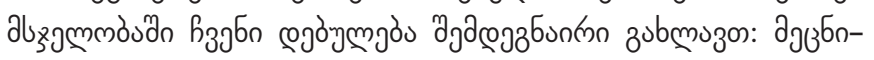

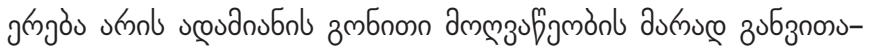

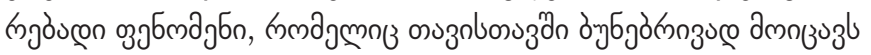

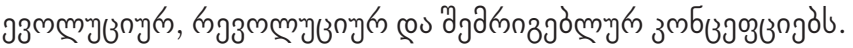

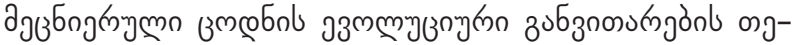

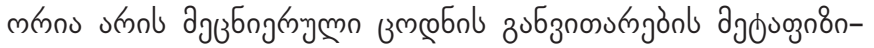

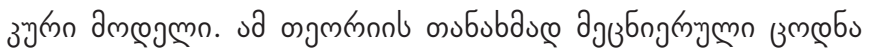

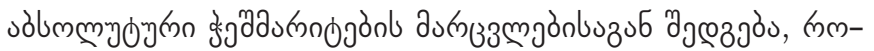

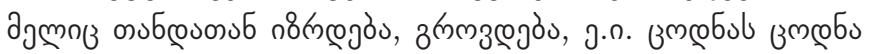

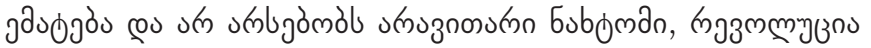

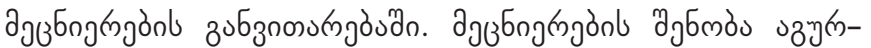

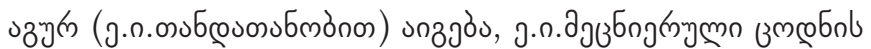

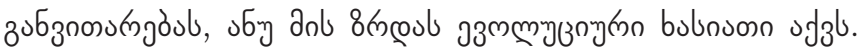
(Avaliani,1986:40).

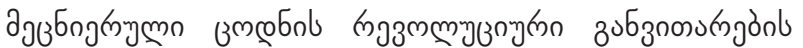

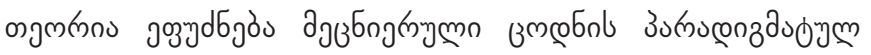

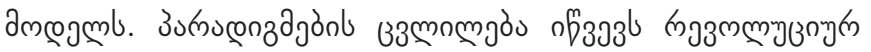

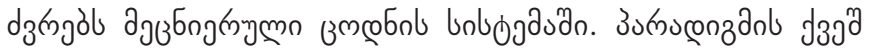

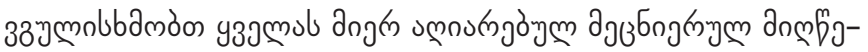

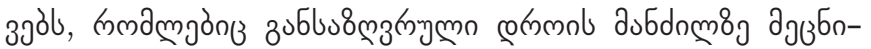

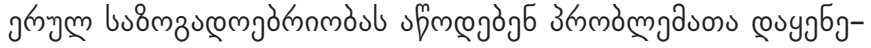

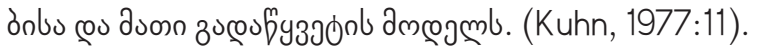

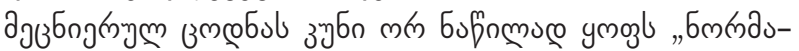

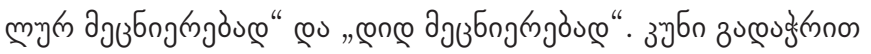

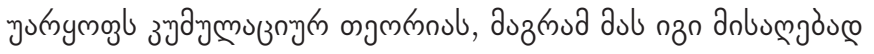

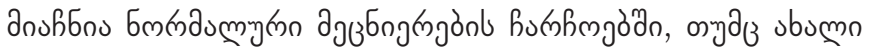

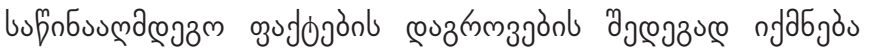

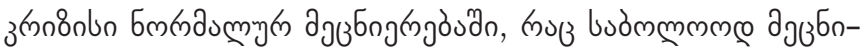

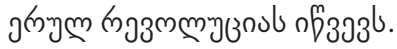

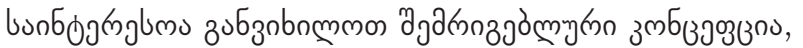

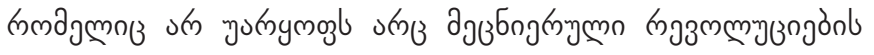

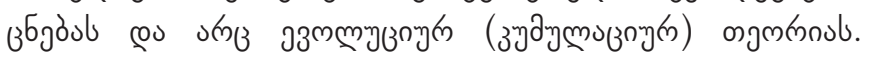

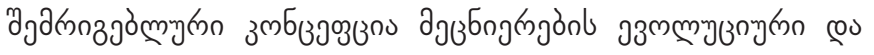

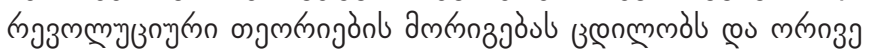

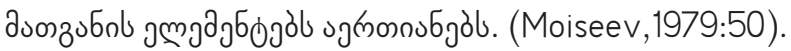

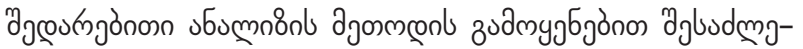

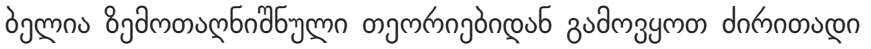

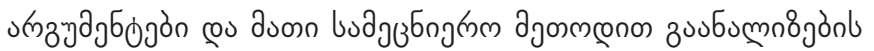

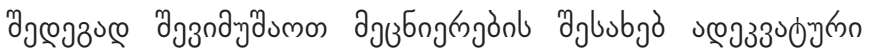

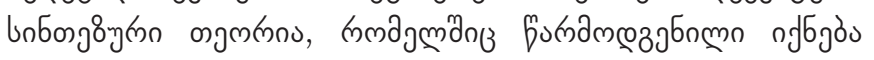

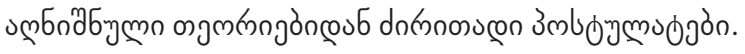

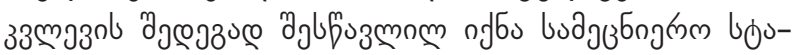

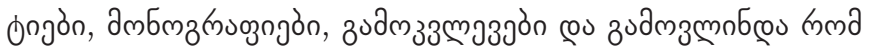

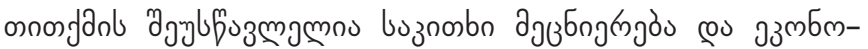

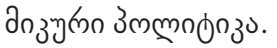

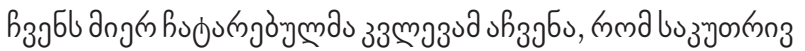

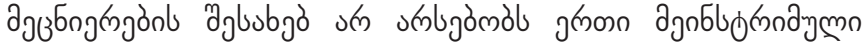

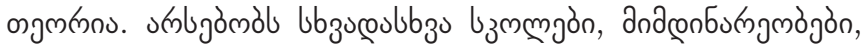

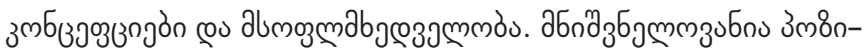

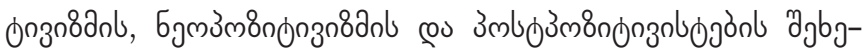

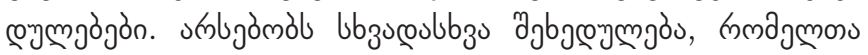

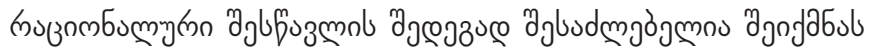

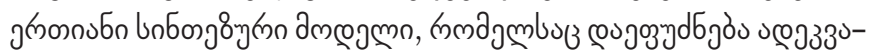

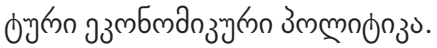

งеэззо

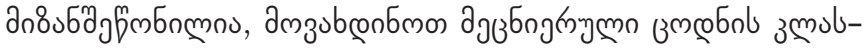
ogmo80uzos.

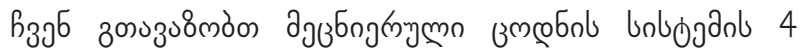

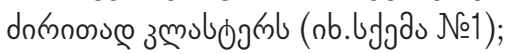

\section{৮ปgว১ №1}

\begin{tabular}{|c|}
\hline 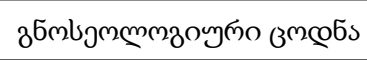 \\
\hline 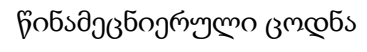 \\
\hline 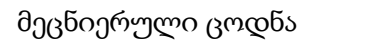 \\
\hline 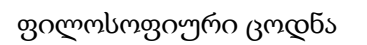 \\
\hline
\end{tabular}

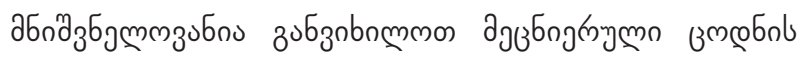

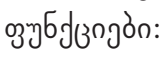

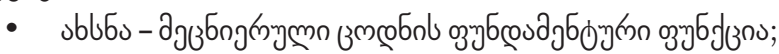

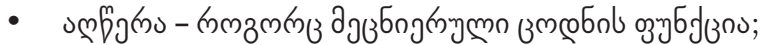

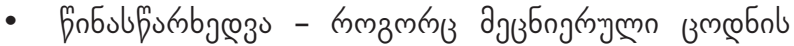

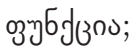

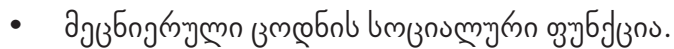

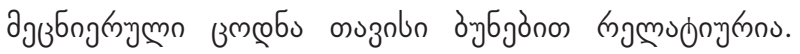

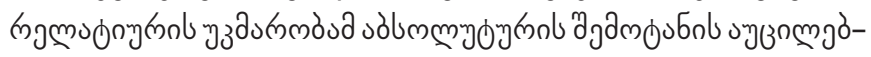

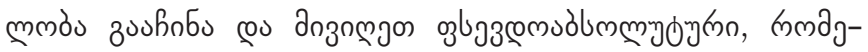

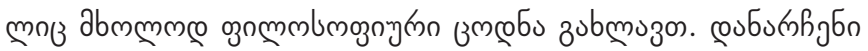

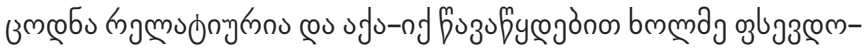

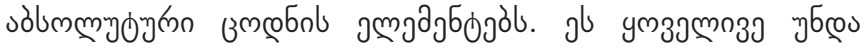

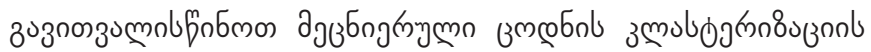

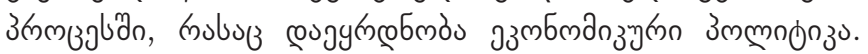

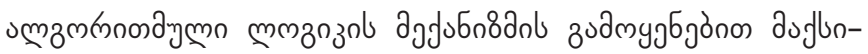

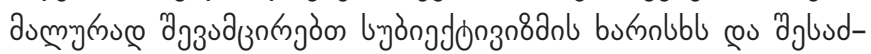

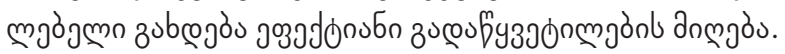

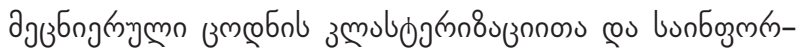

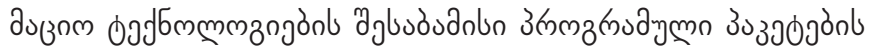

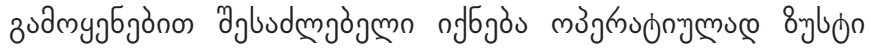

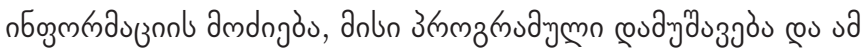

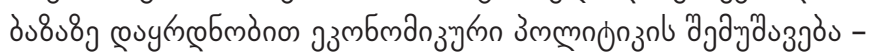
uмbungmajo.

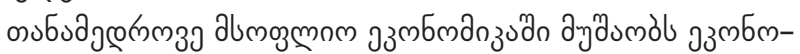

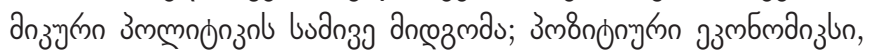

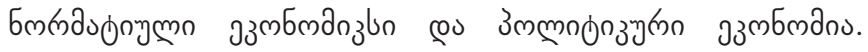
(Query A, 2016:.5-15).

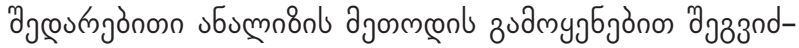

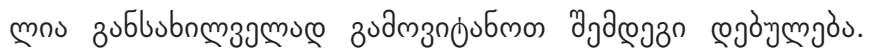




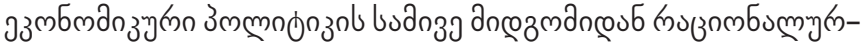

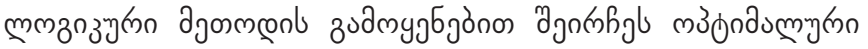

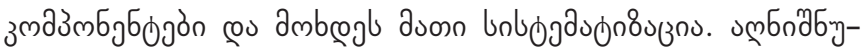

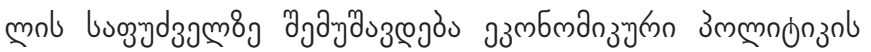

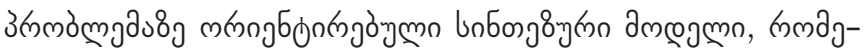

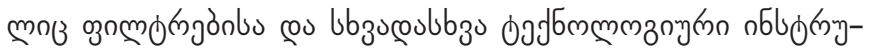

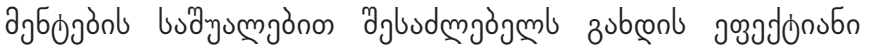

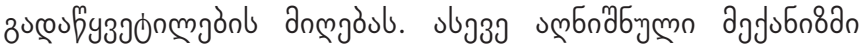

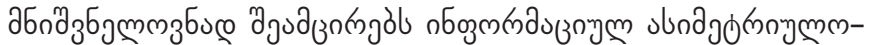

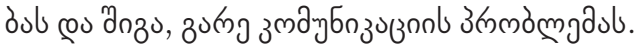

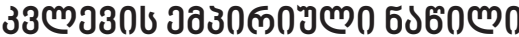

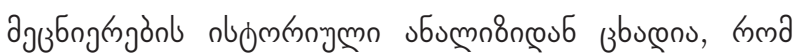

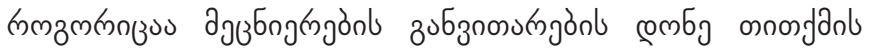

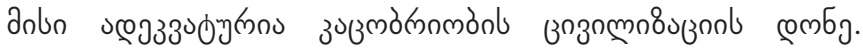

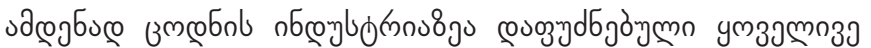

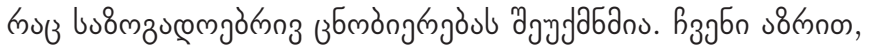

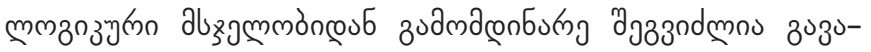

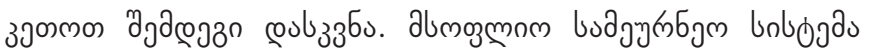

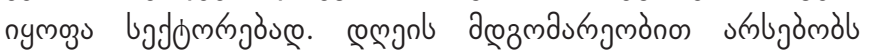
5 bodjom

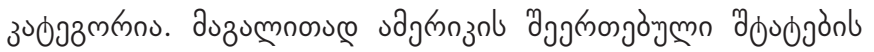

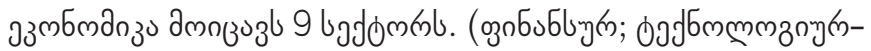

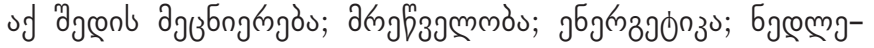

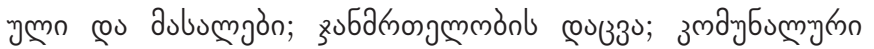

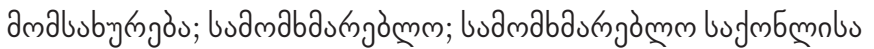

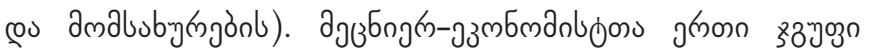

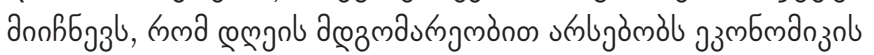
monbn bjdom

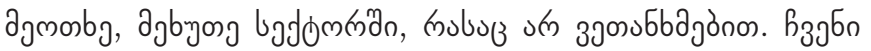

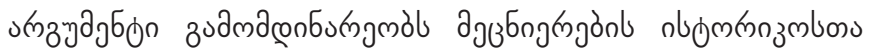

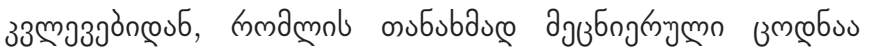

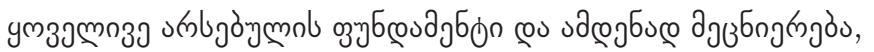

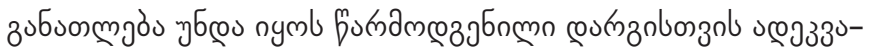

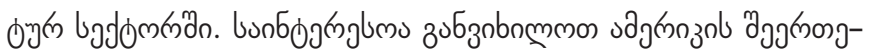

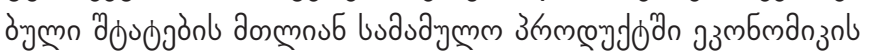

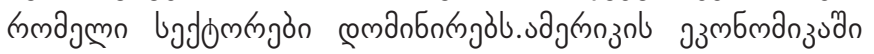

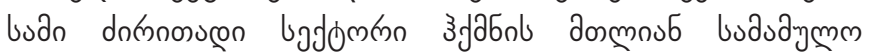

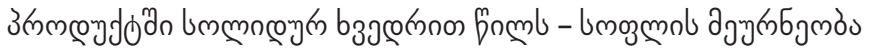

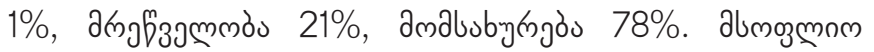

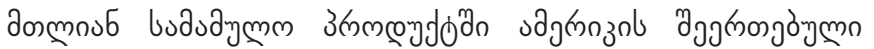

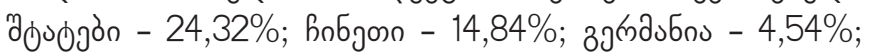

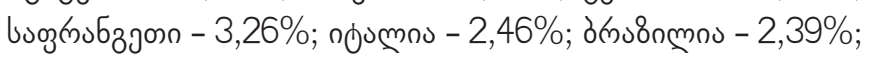

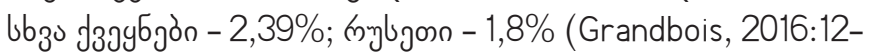
22).

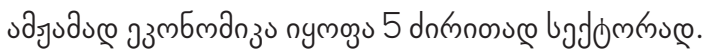

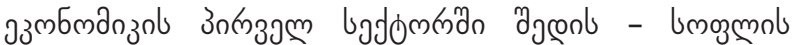

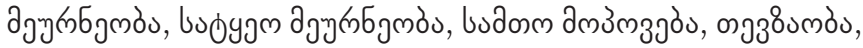
Euconmmòs. эзмбмдази

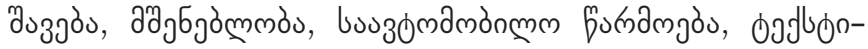

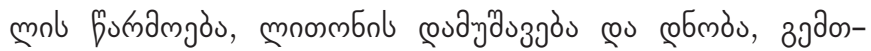

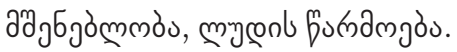

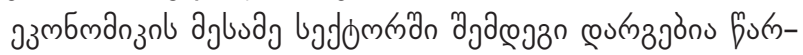

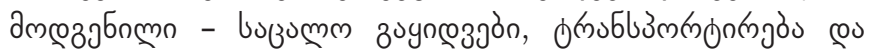

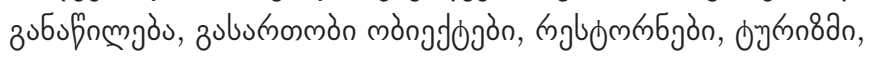

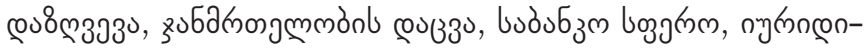

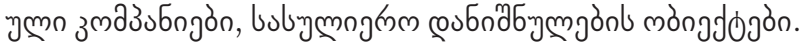

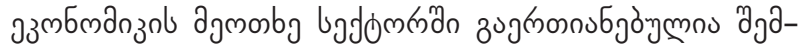

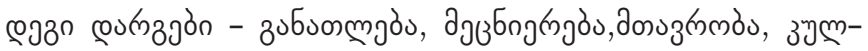

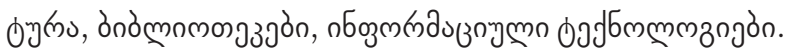

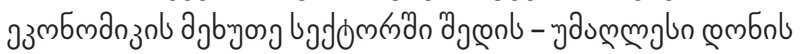

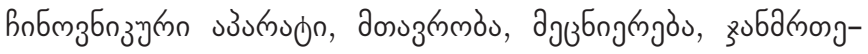

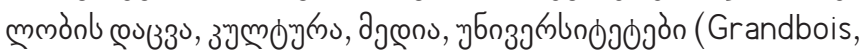
2016:4-15).

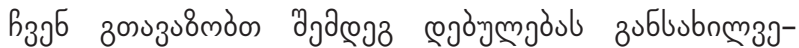

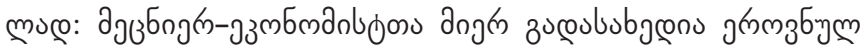

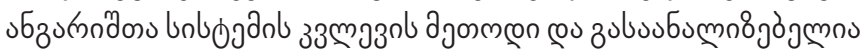

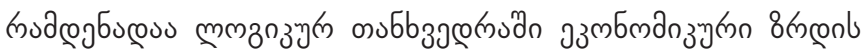

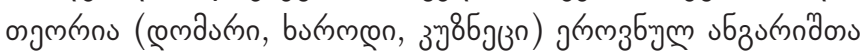

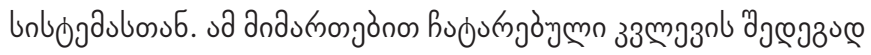

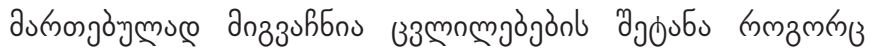

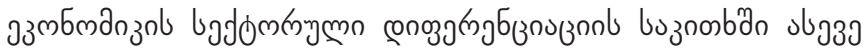

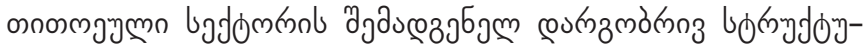

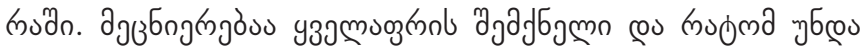

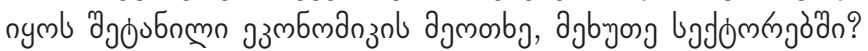

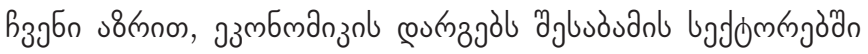

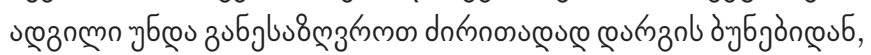

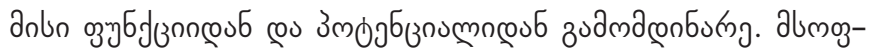

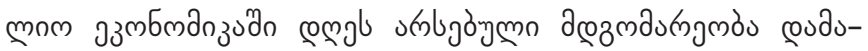

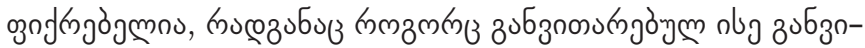

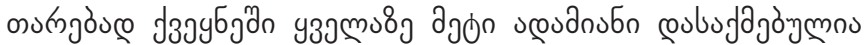
эз з

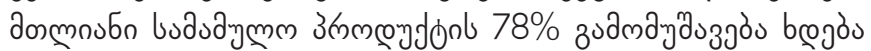
amabubyringòn b udom

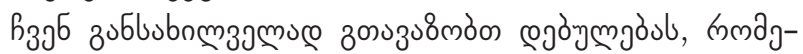

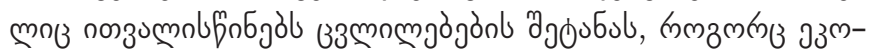

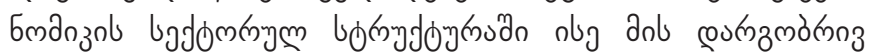

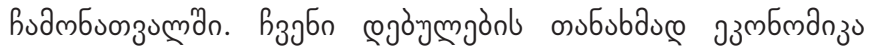

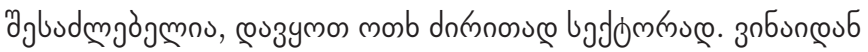

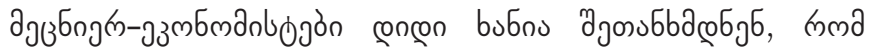

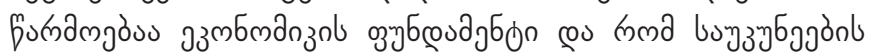

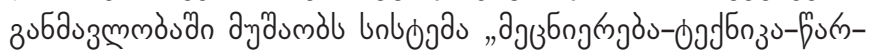

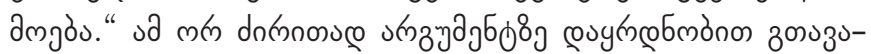

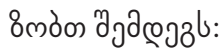

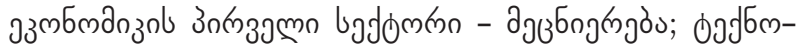

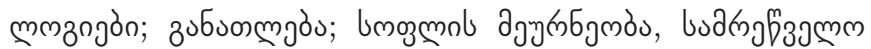

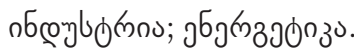

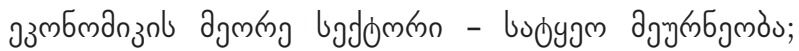




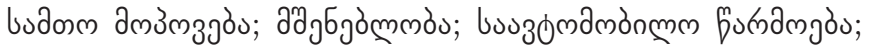

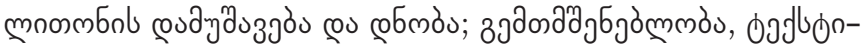

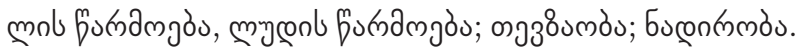

эзмбмдази

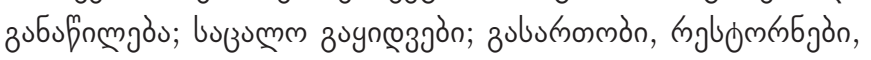

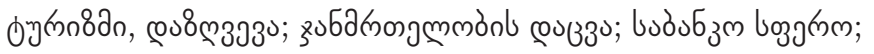

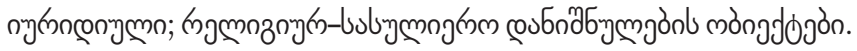

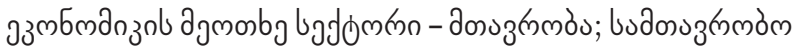

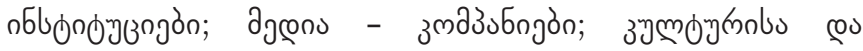

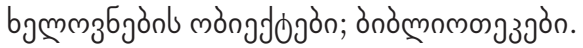

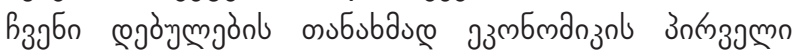

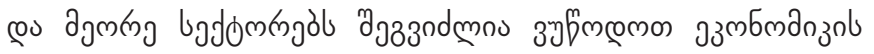

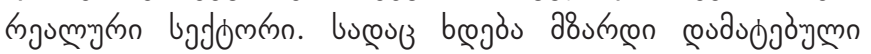

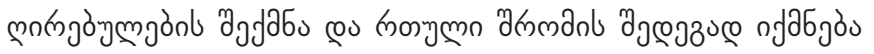

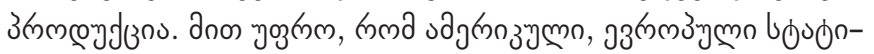

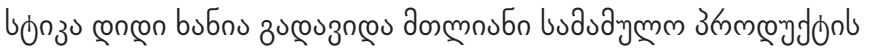

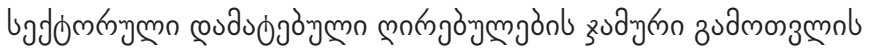
పjomю08 (Lokie, F, 2012:18-22).

วБпәзб

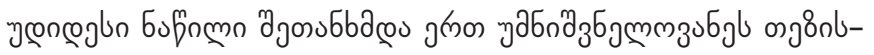

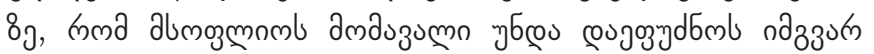

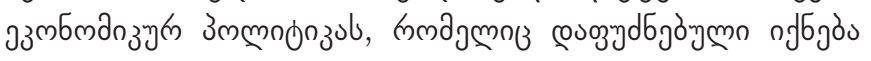

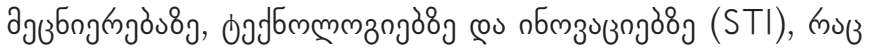

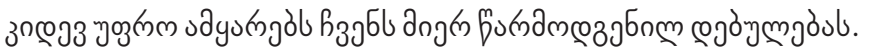
(Meissner, 2013:56-62).

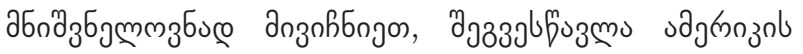

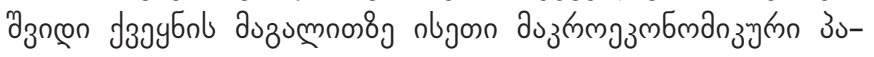

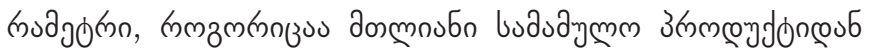

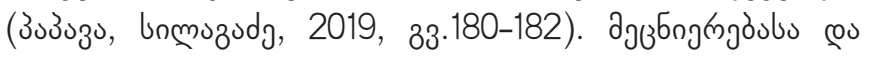

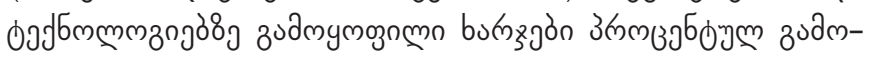

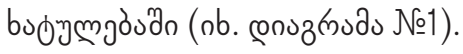

sagm

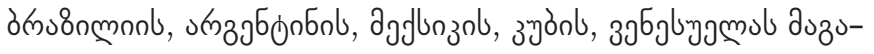

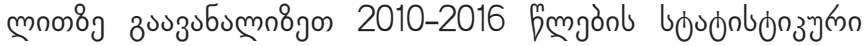

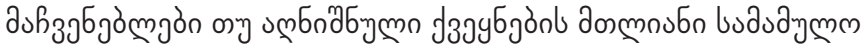

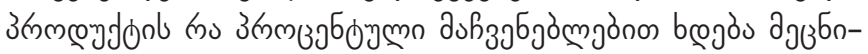

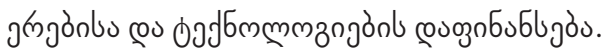

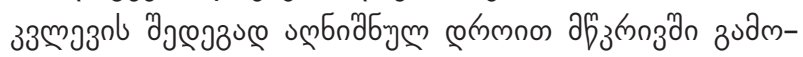

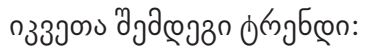

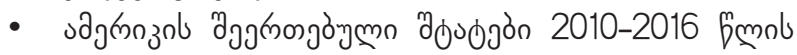

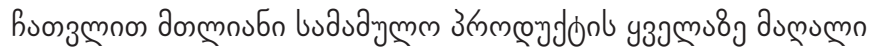
วuศ

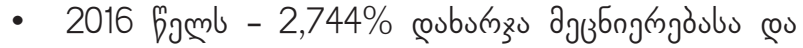

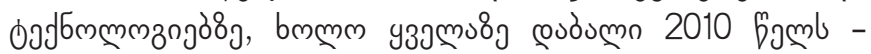
$2,74 \%$;

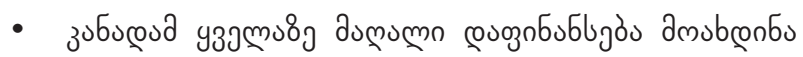

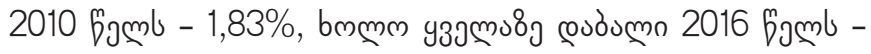
$1,604 \%$

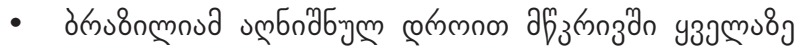

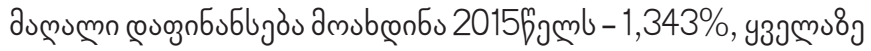

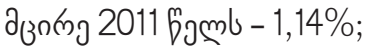

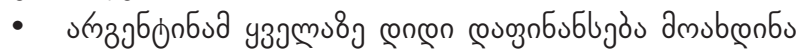

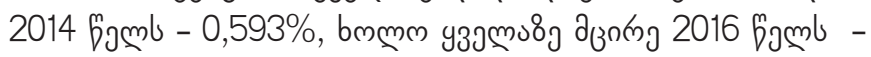
0,533\%;

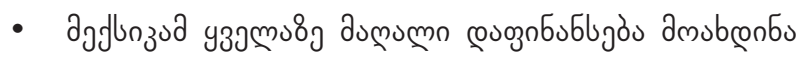

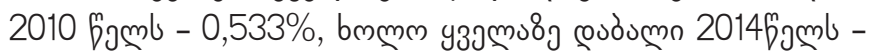
$0,53 \%$;

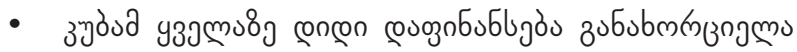

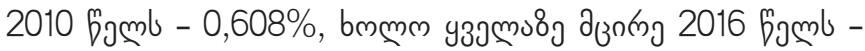
0,349\%;

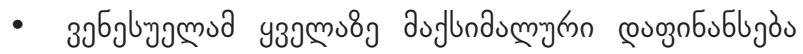

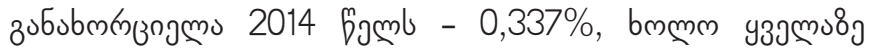

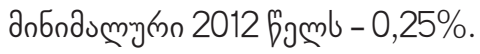

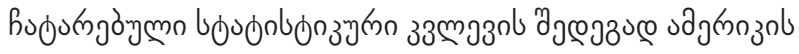

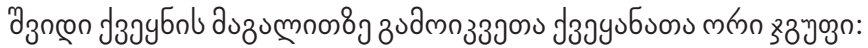

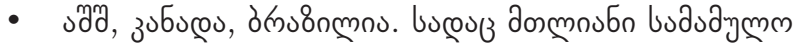

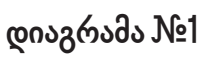

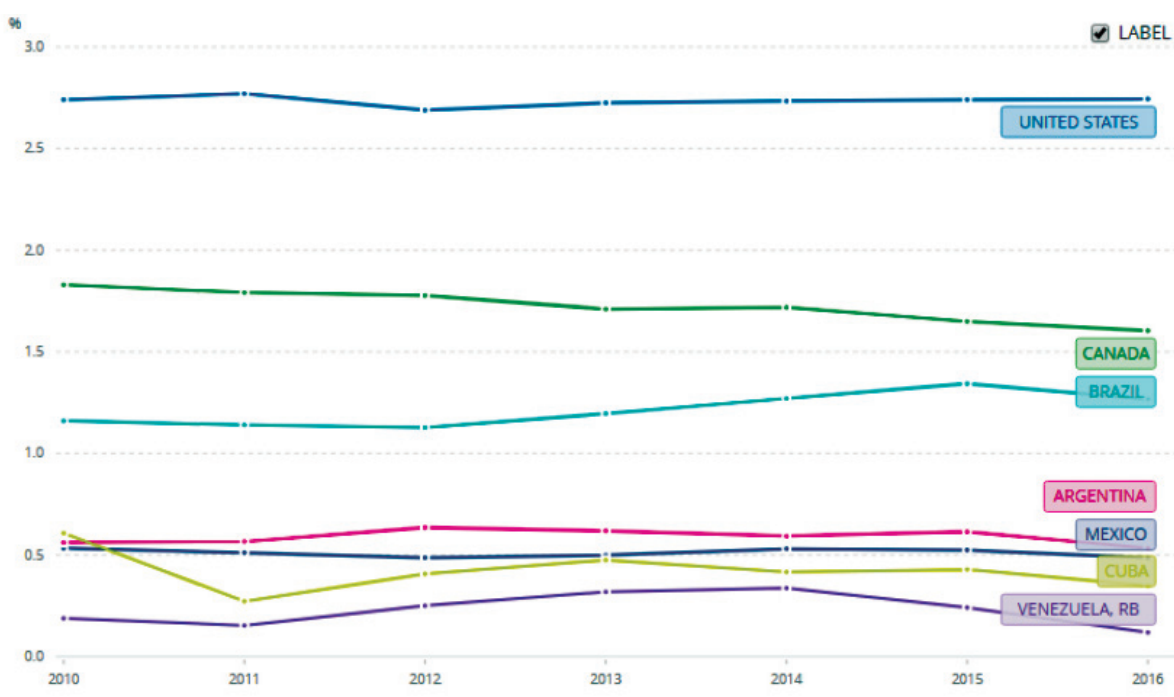

fyurrm: https://data. worldbank.org/topic/science-and-technology?end=2016\&locations=US-CA-MX-AR-BR-VE-CU\&start=2010 


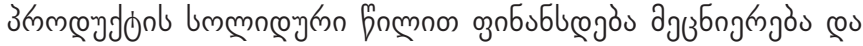

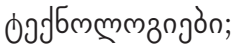

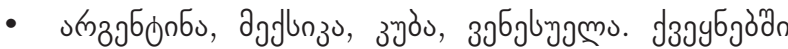
a a mynono.

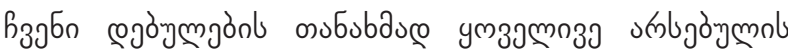

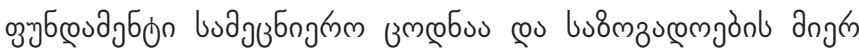

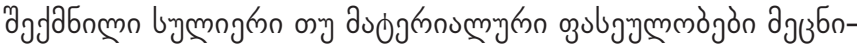

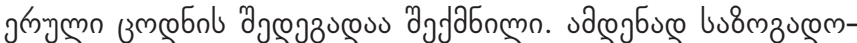

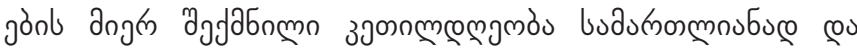

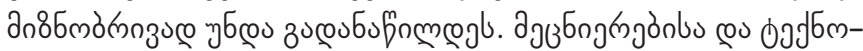

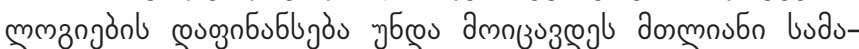

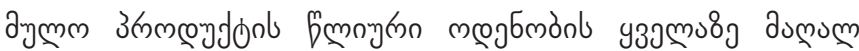

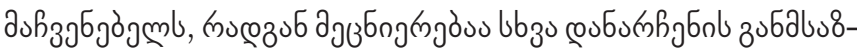

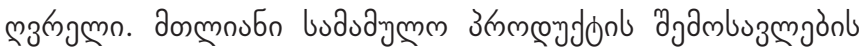

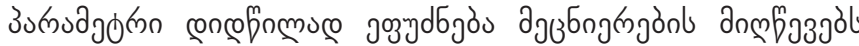

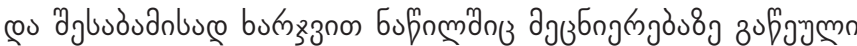

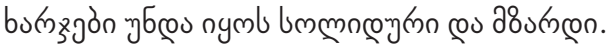

\section{@ob335s}

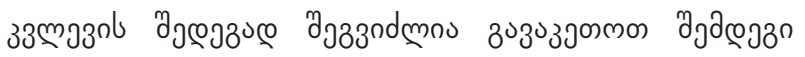

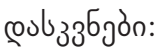

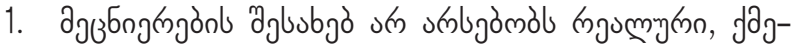

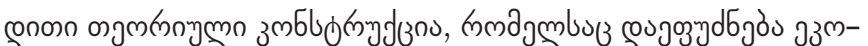

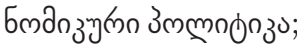

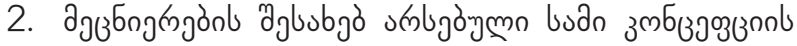

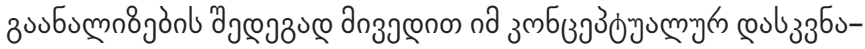

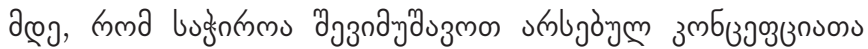

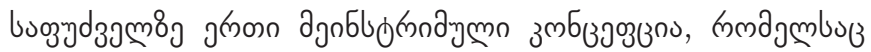

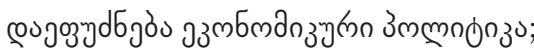

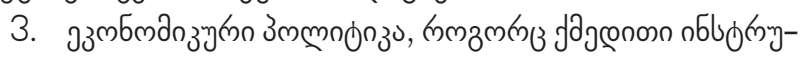

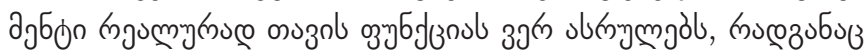

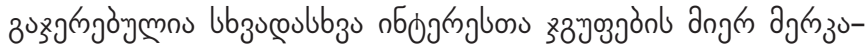

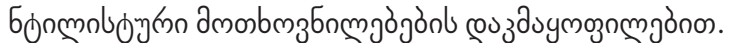

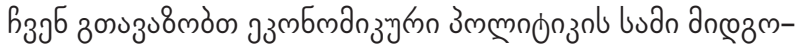

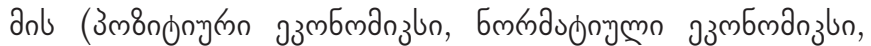

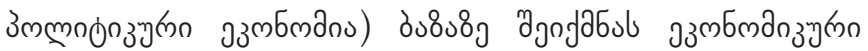

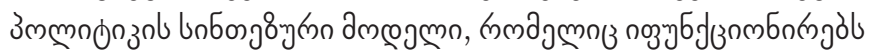

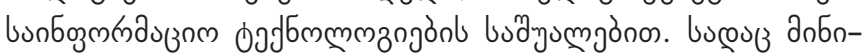

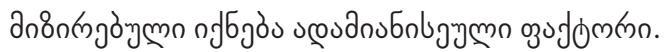

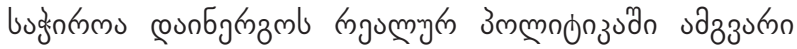

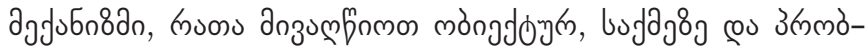

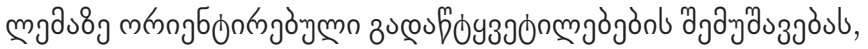

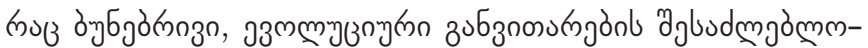
ajal a ajabnl.

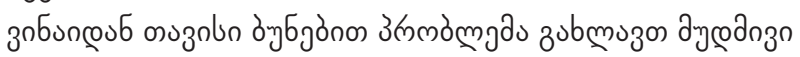

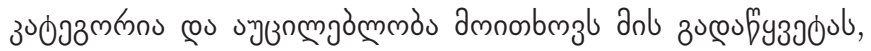

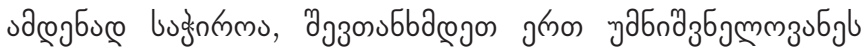

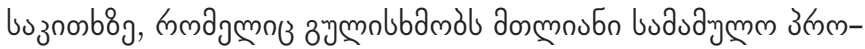

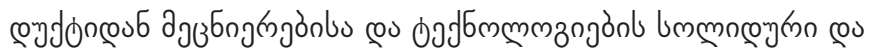
abunnon couogn

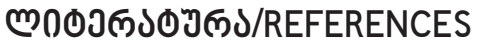

Avaliani, S. (1986).The nature of science and its social purpose.Tbilisi. "Metsniereba” (In Georgian).

Kukania, A. (2006). Science and politics. Tbilisi, "Tobalisi (In Georgian).

Query,A., Kiuri, B, Zhaki ,P., Peri, J.(2016).Ecomomic Policy, Theory and practice.Tbilisi.

"Universitetis gamomtsemloba."(In Georgian).

Lokie F., Blades D.(2015).National accounts system:How to learn and understand.Tbilisi.

"Universitetis gamomtsemloba."(In Georgian).

Papava V., Silagadze A.(2019)." One of the key economic terms-Gross domestic Product- about the Georgian name ".Economics and Business,№1.(In Georgian).

Platoni.(2013).State.Tbilisi."Gamomtsemloba Nekeri".(In Georgian).

Angioni,L.(2016).Aristotle-s definition of scientific knowledge.Canada. „Western University-Canada.“

Barber B.(1994).An Aristocracy of Everyone: The politics of education and the future of America.Oxford "OXFORDUNIVERSITYPRESS."

Granbois Yvonne (2016) In service science and the information professional. Elsevier. www.scienedirect.com

Haldane J. (1923).Daedalus, or science and the future.Cambidge. „Cambidge UNIVERSITY PRESS“.

Meissner D.(2013).Science, Technology and Innovation policy for the Future.Springer.

Russell B.(1994).Icarus or the future of science. California. „CALIFORNIA YNIVERSITY PRESS“.

Bernal, D.(1956). Science in the history of society. Moscow."Inostrannaya literature."(In Russian).

Kuhn, T.(1977).Structure of scientific revolution.Moscow."Progress". (In Russian).

Moisseev, B.(1979).Philosophy and methodology of science,conformity principle. Moscow."Polbu.Ru".(In Russian).

Novik , I.(1963) Cybernetics.Philosophical and sociological problems. Moscow."Gospolitizdat".(From Russian).

Russo, J. (1969).Treatises. Moscow."Nauka".(In Russian). 